\title{
Modelo didáctico de un proceso de producción
}

\section{Teaching model of a production process}

DELGADO-GÓMEZ, Gilberto†, ACOSTA-GONZÁLEZ, Yanid, MUÑOZ-DÍAZ, Ismael y UDAVEDÍAZ, Ma. De Lourdes

Universidad Tecnológica de Aguascalientes, Aguascalientes MX, Blvrd Juan Pablo II, La Cantera, 20200 Exhacienda

ID $1^{\text {er }}$ Autor: Gilberto, Delgado-Gómez / ORC ID: 0000-0001-5213-9432, CVU CONACYT ID: 998195

ID $1^{\text {er }}$ Coautor: Yanid, Acosta-González / ORC ID: 0000-0001-9112-7872, Researcher ID Thomson: S-5620-2018, CVU CONACYT ID: 449264

ID $2^{\text {do }}$ Coautor: Ismael, Muñoz-Díaz / ORC ID: 0000-0003-2779-8932, CVU CONACYT ID: 449264

ID $3^{\text {er }}$ Coautor: Ma. De Lourdes, Udave-Díaz / ORC ID: 0000-0003-4652-6262, CVU CONACYT ID: 998064

DOI: $10.35429 /$ JOTE.2019.9.3.1.7

Recibido 10 de Julio, 2019; Aceptado 27 de Septiembre, 2019

\begin{abstract}
Resumen
Se diseñó un modelo didáctico, para simular condiciones reales de un proceso de producción, a efecto de generar aprendizajes situados relacionados a la carrera de Procesos Industriales, Área Manufactura. Considera una estación de trabajo y un prototipo o producto a fabricar, donde los estudiantes diseñan una línea de producción, en la que hacen "corridas" consecutivas del proceso, que les sirvan para ir aplicando herramientas de Lean manufacturing, pertinentes, hasta lograr "un flujo continuo" de producción; así también se deben garantizar resultados de calidad, y funcionalidad del producto. Los antecedentes que dieron origen a esta propuesta, son "best practices" en empresas de clase mundial particularmente en la industria automotriz, donde se utilizan simuladores para generar competencias tecnicas y metodológicas en su personal administrador de la producción. Para el primer nivel del modelo, se utiliza un prototipo sencillo, construido con 'legos' y se tiene proyectado en el mediano plazo, evolucionarlo a uno de mayor potencial, que incluso sirva para ofrecer entrenamiento al sector productivo. Se realizó un pilotaje con la participación de un grupo de estudiantes, habiendo confirmado la utilidad del mismo, al recrear conocimientos de la asignatura de métodos de trabajo, obteniendo resultados y aprendizajes significativos, que se contienen en el presente artículo.
\end{abstract}

Modelo didáctico, Lean manufacturing, Simulación

\begin{abstract}
A didactic model was designed to simulate real conditions of a production process, in order to generate learning related to the career of Industrial Processes, Manufacturing Area. The model considers a work station and a prototype or product to be manufactured, where the students design a production line, in this work station, they make consecutive "runs" of the process, in order to help them for appling relevant Lean manufacturing tools, until achieving "A continuous flow" of production; thus also must guarantee quality results, and product functionality. The background for this proposal, are "best practices" in worldclass companies, particularly in the automotive industry, where simulators are used to generate technical and methodological competences in their personnel. For the first level of the model, a simple prototype is used, built with 'legos' and the proposal for the medium term, is to evolve it to one of greater potential, which even serves to offer training to the productive sector. Piloting was carried out with the participation of a group of students, having confirmed the usefulness of the model by recreating knowledge of the subject of work methods, obtaining significant results and learning, which are contained in this article.
\end{abstract}

Didatic model, Lean manufacturing, Simulation

Citación: DELGADO-GÓMEZ, Gilberto, ACOSTA-GONZÁLEZ, Yanid, MUÑOZ-DÍAZ, Ismael y UDAVE-DÍAZ, Ma. De Lourdes. Modelo didáctico de un proceso de producción. Revista de Educación Técnica. 2019. 3-9: 1-8.

$\uparrow$ Investigador contribuyendo como primer autor. 


\section{Introducción}

El estado de Aguascalientes, durante el primer trimestre del año 2018, alcanzó uno de los mayores incrementos de su actividad económica con un 5.3\%, contrastado con el mismo periodo de 2017 , en que se tuvo $3.1 \%$ (LJA.MX, 2018). Meade, director de la industria automotriz en ProMéxico, menciona: "no se trata de quien produce más, sino de quien se adapte mejor a las tendencias y condiciones del mercado" (Pineda, 2018);

Estos niveles de crecimiento están estrechamente relacionados con el sector de la manufactura y para sostenerlos o incluso aspirar a mejorarlos aún más, se debe considerar como pilar fundamental la incorporación de personal técnico y profesional con las competencias pertinentes, que optimicen los resultados de dicha industria.

Por lo tanto, será necesario que las instituciones educativas, adecúen sus estrategias de enseñanza-aprendizaje, con los esquemas y estándares de la industria de manufactura local, considerando que una alta proporción de la misma, pertenece directa o indirectamente al sector automotriz y de autopartes, donde se opera con sistemas de manufactura avanzada (just in time, estandarización, lean manufacturing, etc.).

Una opción para el desarrollo de competencias alineadas a las requeridas en los procesos productivos, es la de utilizar simuladores, donde se puedan replicar variables y condiciones típicas y reales de los procesos, ofreciendo la oportunidad a los estudiantes de adquirir aprendizajes situados, que les permita adquirir competencias y tomar decisiones en la mejora de los procesos productivos.

Algunas experiencias de instituciones educativas para ir consiguiendo esta afinidad de programas de estudio con situaciones reales de los sistemas productivos, se mencionan a continuación:

De acuerdo a Gastélum-González, (2004), diseñó un equipo para el laboratorio de Ingeniería Industrial, con el objetivo de considerar los principios del diseño de estaciones de trabajo que promuevan las posturas naturales del cuerpo, complementando la teoría vista en clases.
Peláez_Mejía, Payán-Quevedo \& Salazar-Ramos, (2015), de la Universidad del Valle sede Buga, aplicaron la metodología Bucket Brigades (BB picking), como una introducción del Toyota Sewn Products Management System de ensamble, desensamble o de Manufactura que trata de balancear una línea, buscando lograr la asignación óptima de los recursos obteniendo mejoras en el desempeño de la misma.

La consultoria QCDSM training, desarrollo un modelo para simular las condiciones reales de un proceso de fabricación, y donde se van incorporando herramientas de mejora continua en diferentes niveles de la organización, así como al propio proceso productivo, evento que se replica en diferentes países de Europa, América Latina, particularmente Estados Unidos y Australia (QCD Systems Inc. Website by Liquid Scope NZ, 2015).

En en año 2017, realizaron un estudio Tejada-Díaz, Gisbert-Soler, \& Pérez-Molina, sobre el estudio de tiempo y movimiento enfocado al General Sewing Data (GSD), mencionan que es una técnica de gran ayuda para las empresas para mejorar la productividad, reducir los movimientos ineficientes, así como los costos del mismo. Aunque esta metodología va muy específica a operaciones de tipo textil, es decir al corte, costura, planchado, inspección y empaque para mejorar los procesos, requiere que las personas dominen la técnica de la labor que se va a estudiar.

En la Universidad Tecnológica de Aguascalientes, específicamente en la carrera de Procesos Industriales área Manufactura, se ha desarrollado un prototipo que considera una estación de trabajo, con el propósito que los estudiantes adquieran conocimiento y habilidad básicos en la administración de procesos productivos propios del sector manufacturero; el mismo prototipo, también podría utilizarse en mejorar competencias similares en personal trabajador de las propias empresas.

La competitividad empresarial demanda invariablemente cumplir metas relacionadas con: los tiempos de entrega del producto, la garantía de calidad y un costo competitivo; cada una de ellas, genera áreas de oportunidad que el prototipo en desarrollo, puede simular. 


\section{Tiempos entrega del producto}

1. Flujo del proceso.

2. Distribución de operaciones.

3. Balanceo de líneas de producción.

4. Estandarización.

\section{Garantía de Calidad}

1. Especificaciones de materiales.

2. Funcionalidad.

3. Especificaciones de productos.

4. Métodos de inspección.

5. Mediciones y tolerancias.

6. Instrumentos de medición.

7. Ciclo de mejora continua.

8. Kaizén.

9. Poka yoke.

10. Parámetros de control de proceso.

\section{Costo competitivo}

1. Probar nuevos métodos de trabajo.

2. Desperdicio de material.

3. Inventarios.

4. Logística y flujo de materiales.

\section{Resultados esperados}

1. Diseño de producto.

2. Diseño de proceso.

a. Secuencia de operaciones.

b. Medición del trabajo.

c. Eficiencia.

3. Puntos de control de calidad.

\section{Balanceo de la línea.}

5. Capacidad de producción.

6. Funcionalidad de producto.

\section{Propuesta}

Desarrollar un prototipo que simule las condiciones de una línea de producción y por lo tanto, ayude a recrear las variables de entrada, de proceso y de salida del mismo; este proyecto debe beneficiar tanto a estudiantes de la carrera de procesos industriales, como a empresas del sector productivo local, implementando mejoras, mediciones, tiempos y movimientos del trabajo, balanceos de línea entre otros.

\section{Hipótesis o supuestos}

Si se cuenta con una estación de trabajo para simulación de procesos, docentes y alumnos de la UTA, tendrán una herramienta efectiva de apoyo a la consolidación de sus competencias para integrarse proactivamente en el sector productivo.

\section{Justificación}

La demanda de resultados en la industria manufacturera genera una fuerte competitividad, y en consecuencia se deben estar mejorando continuamente los resultados de tiempos de entrega, calidad y costos, apoyados en la formación de las plantillas de personal.

Alumnos y trabajadores del sector productivo adquieran las competencias para intervenir en los procesos productivos.

\section{Metodología}

Investigación descriptiva se refiere a ver los resultados que se van obteniendo, para ir iterando ajustes y alcanzar un objetivo de funcionamiento $\mathrm{o}$ de resultado. Su consideración en el proyecto aplica, al hacer corridas secuenciales de producción en la estación de trabajo, y que tanto los alumnos como con el personal de empresas manufactureras, encuentren áreas de oportunidad para ir perfeccionando consistentemente las prácticas y estándares a utilizar (metodología Deming). 
El desarrollo del modelo didáctico o prototipo, se planteó para tres momentos o etapas:

a) La primera es el diseño del producto: partes, sub-ensambles y línea final.

b) La segunda etapa, es el diseño del proceso: estaciones, secuencia de operaciones, estándares de trabajo, tiempo estándar y tiempo ciclo de cada una.

c) La tercera etapa: es la simulación que consiste en una serie de "corridas del proceso" en que se monitorean las variables del mismo, desde la organización de las estaciones de trabajo, el flujo, la estandarización de operaciones, el balanceo de la línea, así también los resultados esperados particularmente la confirmación de la capacidad.

\section{Aplicación en campo}

A continuación, se muestran resultados, mejoras y aprendizajes obtenidos en la prueba piloto, que realizó un grupo de estudiantes de la carrera de Procesos Industriales Área Manufactura.

\section{Objetivo}

Simular las variables de control de un proceso de manufactura, utilizando el modelo didáctico en desarrollo.

\section{Objetivos específicos}

1. Identificar los productos.

2. Identificar los procesos.

3. Diseñar la estación de trabajo.

4. Hacer las corridas de prueba.

Se definió un área aproximadamente de 50 metros cuadrados dentro del taller 2, de la Universidad Tecnológica de Aguascalientes, para instalar el modelo didáctico (ver Figura 1).

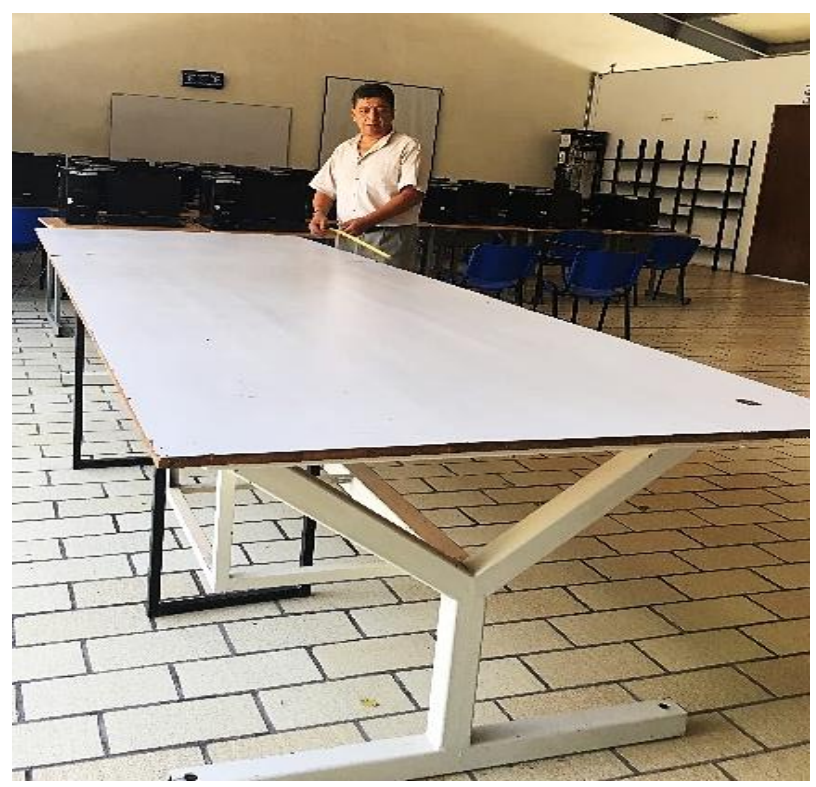

Figura 1 Definición del área de trabajo

Se utilizó una mesa con dimensiones de 3.65 mts. x 1.20 mts. como zona para instalar la línea de producción, delimitando en ella las áreas de recibo, operación y almacén de producto terminado (ver Figura 2).

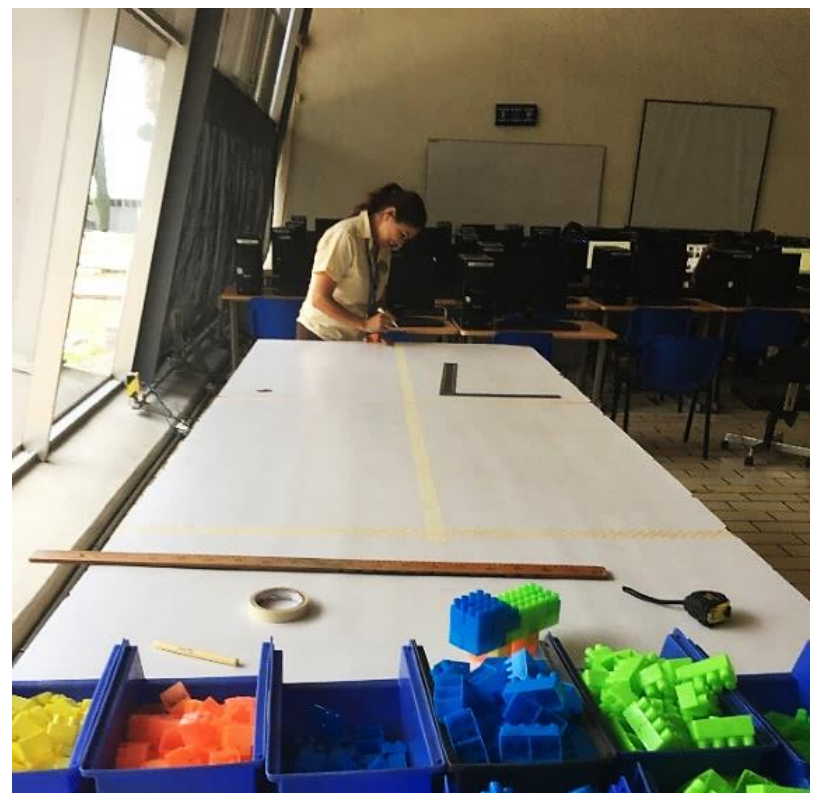

Figura 2 Delimitación de la estación de trabajo

\section{Prototipo a fabricar}

Se estableció un prototipo de producto, armado con "legos" cabe mencionar que este será un prototipo de transición, ya que se tiene el plan de posteriormente mejorarlo utilizando materiales que permitan especificaciones más exactas, tolerancias, pruebas de funcionalidad etcétera; el prototipo consiste en un camión utilitario tipo cab-star, compuesto por: tres subensambles (cabina, chasis, llantas) y el ensamble de conjunto final (ver Figura 3). 


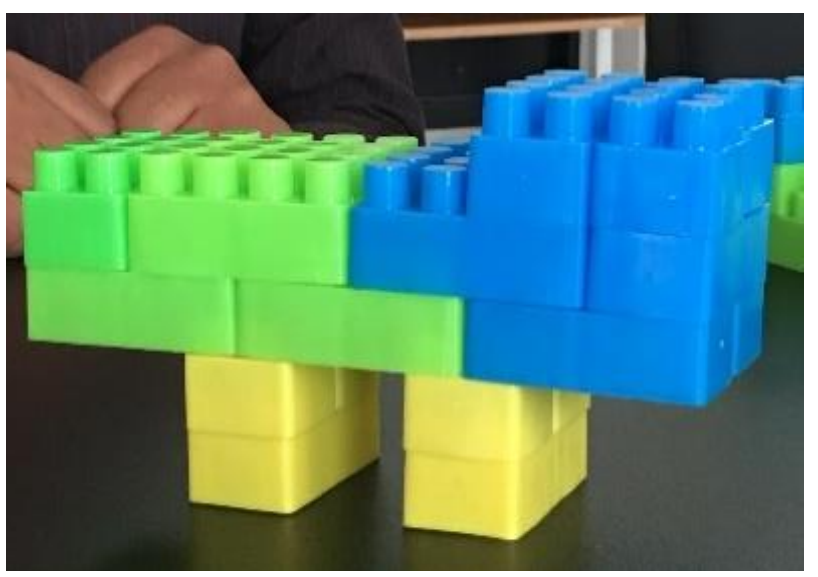

Figura 3 Prototipo a diseñar por parte de los estudiantes

El grupo de trabajo, recibió un requerimiento de fabricación de una cantidad determinada de unidades, como único insumo para generar el diseño del proceso, definieran la cantidad de estaciones de trabajo, la secuencia de fabricación, los estándares para cada subensamble, así como los tiempos ciclo y tacto, para determinar la capacidad de fabricación. El pilotaje consiste en varias corridas secuenciales hasta estabilizar el proceso.

\section{Resultados}

Los estudiantes generaron un diseño de proceso (ver Figura 4); posteriormente realizaron varias corridas para cumplir con el requerimiento de cliente, para ello fueron secuencialmente determinando tiempos ciclo para cada componente, identificando cuellos de botella para alcanzar el flujo continuo, asimismo hicieron mejoras en ergonomía y documentaron su kaizen.

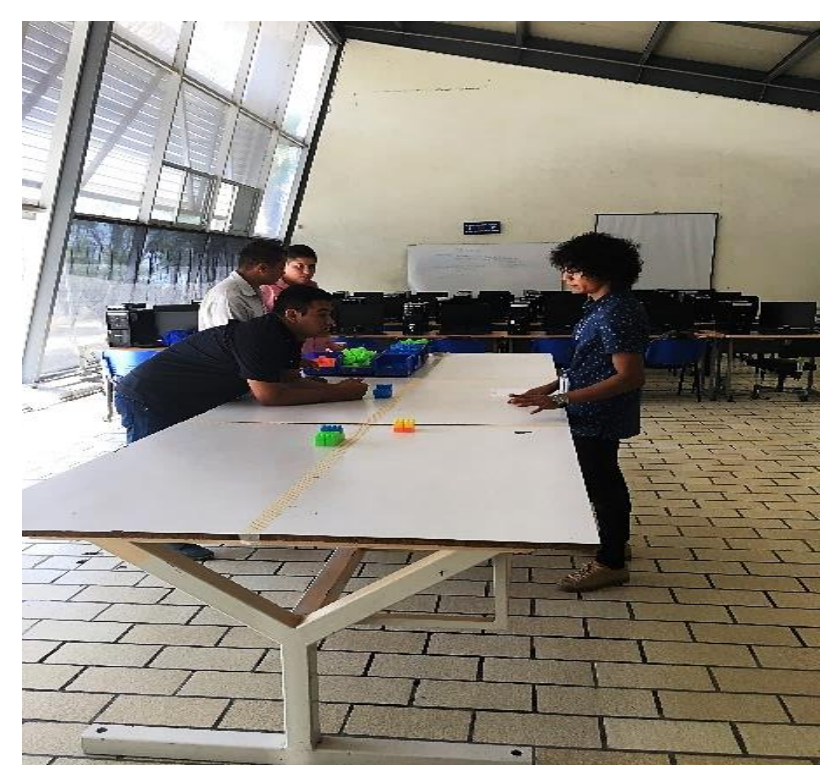

Figura 4 Organización de las estaciones de trabajo
Primero, diseñaron la secuencia del proceso y el elaboraron el diagrama de flujo del mismo (ver Figura 5).

\begin{tabular}{|l|c|l|l|l|}
\hline \multicolumn{1}{|c|}{ Operación } & & & V & D \\
\hline Ensamble de base de carroceria A & & & & \\
\hline Ensamble de base de carroceria B & & & & \\
\hline Ensamble de base de carroceria C & & & & \\
\hline Ensamble de parte superior de carroceria A & & & & \\
\hline Ensamble de parte superior de carroceria B & & & & \\
\hline Ensamble de torreta de cabina & & & & \\
\hline Ensamble de llantas A & & & & \\
\hline Ensamble de llantas B & & & & \\
\hline Inspección final & & & & \\
\hline Liberar material & & & & \\
\hline
\end{tabular}

Figura 5 Diagrama de flujo del ensamble del carro

Fuente: elaboración realizada por los alumnos de Procesos Industriales

Consideraron 10 elementos para el ensamble del prototipo y realizaron 12 corridas del mismo (ver Figura 6).

\begin{tabular}{|c|c|c|c|c|c|c|c|c|c|c|c|c|c|c|}
\hline Operación & 1 & & 3 & 4 & & 6 & & & 8 & & 10 & 11 & 12 & \\
\hline Ensamble de base de carroceria $\mathrm{A}$ & 0.6 & 0.5 & 0 & 0.6 & $\begin{array}{c}60.6 \\
6\end{array}$ & 0. & & & 0.60 & 0.7 & 0.5 & 0.6 & 0.6 & 0.6 \\
\hline Ensamble de base de carroceria B & 0.6 & 0.7 & 0. & 0.7 & 70.6 & 0. & & & 0.6 & 0.6 & 0.6 & 0.6 & 0.6 & 0.625 \\
\hline Ensamble de base de carroceria C & 0.6 & 0.5 & 0. & 0.5 & 50.6 & 0. & & & 0.60 & 0.6 & 0.6 & 0.6 & & 0.583333 \\
\hline Ensamble de pate superior de carroceria A & 1.5 & 1.2 & 1. & 1.3 & \begin{tabular}{|l|l|}
3 & 1.3 \\
\end{tabular} & 1. & & & 1.5 & 1.5 & 1.5 & 1.5 & 1.51 & 1.408333 \\
\hline Ensamble de pate superior de carroceria B & 1.1 & 1.1 & 1. & 1.3 & \begin{tabular}{|l|l|l}
3 & 1.1 \\
\end{tabular} & 1. & & & 1.1 & 1.1 & 1.1 & 1.1 & 1.1 & 1.125 \\
\hline \begin{tabular}{|l|l} 
Ensamble de torreta de cabina \\
\end{tabular} & 0.8 & 0.9 & 0. & 0.7 & 0.7 & 0. & & 8.8 & 0.8 & 0.7 & 0.7 & 0.8 & & 0.766667 \\
\hline Ensamble de lantas A & 0.9 & 0.9 & 0. & 0.9 & 90.9 & 0 & & .9 & 0.9 & & 0.9 & 0.9 & & 0.908333 \\
\hline Ensamble de lantas B & 0.6 & 0.7 & 0 & 0.6 & $\begin{array}{ll}6.6 \\
6.6\end{array}$ & 0 & & 7 & 0.7 & 0.6 & 0.6 & 0.6 & & 0.633333 \\
\hline Inspeccion final & 0.5 & 0.5 & 0. & 0.5 & \begin{tabular}{|l}
50.5 \\
0.5
\end{tabular} & 0 & & 6 & 0.6 & 0.6 & 0.6 & 0.6 & 0.50 & 0.541667 \\
\hline Lberar material & 0.5 & 0.5 & 0. & 0.6 & \begin{tabular}{|l|l}
6 & 0.5 \\
\end{tabular} & & & 6 & 0.6 & 0.7 & 0.6 & 0.6 & 0.6 & 0.575 \\
\hline & & & & & & & & & & & & & & 7.766667 \\
\hline
\end{tabular}

Figura 6 Toma de tiempos de la simulación

Fuente: elaboración realizada por los alumnos de Procesos Industriales

Se determinaron los factores de actuación para el tiempo ciclo que son: el esfuerzo, la habilidad, el ambiente y la consistencia, con un total del $10 \%$ y para el tiempo estándar los suplementos de descanso de acuerdo al Instituto de Administración Científica de las Empresas del 9\% de paros programados del $6.25 \%$ (Ver Tabla 1).

\begin{tabular}{|c|c|}
\hline Factores de actuación & Suplementos \\
\hline $\begin{array}{l}\text { Habilidad: Malo_ } 10 \% \\
\text { Esfuerzo: Medio_- } 0 \% \\
\text { Condiciones: Media } \\
\text { Consistencia: Mala__ } 0 \%\end{array}$ & $\begin{array}{l}\text { Hombre } \\
\text { Trabaja de pie_2 } 2 \% \\
\text { Ruido intermitente y } \\
\text { fuerte_2\% } \\
\text { Necesidades } \\
\text { personales__5\% } \\
\text { Paros programados } \\
\text { Comida }=6.25 \%\end{array}$ \\
\hline
\end{tabular}

Tabla 1 Factores de actuación y suplementos para el estudio de trabajo

Fuente: elaboración realizada por los alumnos de Procesos Industriales

DELGADO-GÓMEZ, Gilberto, ACOSTA-GONZÁLEZ, Yanid, MUÑOZ-DÍAZ, Ismael y UDAVE-DÍAZ, Ma. De Lourdes. Modelo didáctico de un proceso de producción. Revista de Educación Técnica. 2019 
El resultado obtenido en el tiempo ciclo fue de 8 segundos/pieza. Para el tiempo estándar se tuvo como resultado 9 segundos/pieza (Ver Tabla 2)

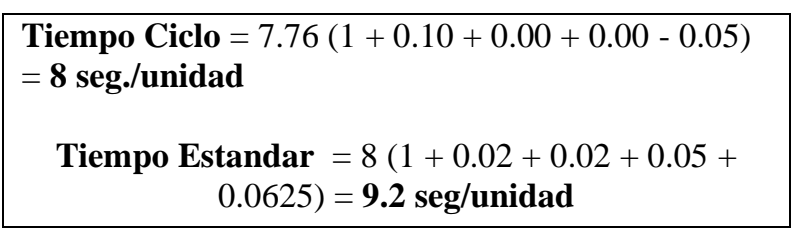

Tabla 2 Tiempo ciclo y tiempo estándar del prototipo didáctico

Fuente: elaboración realizada por los alumnos de Procesos Industriales

Con base al resultado de tiempos del proceso, determinaron las capacidades: capacidad nominal de 600 unidades/jornada; capacidad efectiva de 562 unidades /jornada (Ver Tabla 3).

\begin{tabular}{|c|c|c|c|}
\hline & Hora & Turno & Semanal \\
\hline $\begin{array}{l}\text { Capacidad } \\
\text { Nominal }\end{array}$ & 75 & 600 & 3600 \\
\hline $\begin{array}{l}\text { Capacidad } \\
\text { Efectiva }\end{array}$ & 70 & 562 & 3372 \\
\hline Capacidad Real & 59 & 474 & 2844 \\
\hline
\end{tabular}

Tabla 3 Estudio de capacidades de la estación de trabajo Fuente: elaboración realizada por los alumnos de Procesos Industriales

Durante este pilotaje se realizaron mejoras, logrando una reducción del número de 10 elementos originalmente establecidos a 6 (incluyendo una inspección) (Ver Tabla 4).

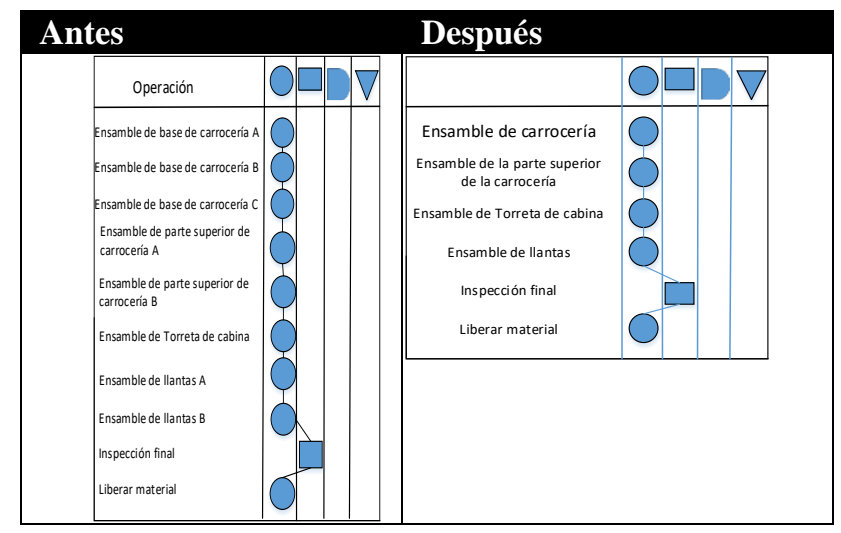

Tabla 4 Kaizen de la estación de trabajo Fuente: elaboración realizada por los alumnos de Procesos Industriales

\section{Conclusiones}

Con esta simulación el grupo de estudiantes, relacionó a una aplicación real, los conocimientos adquiridos en la materia de métodos y sistemas de trabajo como, balanceo de la línea, tiempo ciclo y estándar, entre otros temas, con lo cual se fortalecen las competencias establecidas para la carrera de procesos industriales, área manufactura.

Otro aprendizaje observado, corresponde a las habilidades de trabajo colaborativo, ya que, al inicio del pilotaje, hubo dificultades de comunicación y coordinación, factores que fueron superados, logrando el objetivo de simular el proceso y generar mejoras. Los participantes percibieron el valor agregado al proceso de enseñanza-aprendizaje que una simulación de esta naturaleza les pueda aportar y comparten sus opiniones en el anexo 1. El modelo se direcciona para recrear en la práctica, conocimientos adquiridos en las asignaturas de especialidad, dentro de la carrera de Procesos Industriales. A efecto de consolidar el modelo didáctico, el prototipo se deberá escalar en su diseño, materiales de fabricación y tolerancias, para fortalecer las prácticas y puedan reflejarse también condiciones de calidad y funcionalidad. Con ello, el modelo tendrá especificaciones que lo hagan atractivo como un recurso de entrenamiento, para el sector productivo.

\section{Anexo}

\section{Anexo 1}

"Al realizar este proyecto logramos identificar que es necesario determinar bien las operaciones con las que se van a trabajar.... para poder determinar un número adecuado de operadores es necesario basarse en el tack time " María Fernanda Hernández De Loera

"En este proyecto descubrí y aprendí como es que se crea una línea de producción desde el principio y la distribución que esta necesita: aunque sea a menor escala siento que me servirá como una buena base para un futuro. También descubrí la importancia del ahorro de mano de obra, ya que se puede ahorrar tiempo y dinero con una buena distribución y un buen balanceo de la línea" Armando Vera Díaz. 
"Siento que esta práctica me sirvió para aprender más de manera didáctica la utilización de las fórmulas para ahorro de operadores, toma de tiempos y el balanceo de la línea en general" Luis Alberto Sánchez.

“... al realizar junto a mis compañeros de trabajo la práctica, puede tener una noción de cómo es organizar una línea, desde la organización que tuvimos con el equipo de trabajo, hasta cuando había que hacer cálculos y pedir informes de tiempos, mediciones, etc."

"Siento que es un buen proyecto y lo utilizare como aprendizaje para realizar la medición de tack times, tiempos ciclos y otras cuantas fórmulas que viví en la teoría y pasé a aplicarlas en la vida real con esta mini línea que me deja un buen aprendizaje" Alejandro Hernández.

\section{Referencias}

Gastélum-González, H. (2004). Centro de Trabajo Multifuncional. Memorias de VI Congreso Internacional de Ergonomía, 89-98.

LJA.MX. (28 de 07 de 2017). Aguascalientes registra crecimiento económico sostenido. Recuperado el 29 de 08 de 2018, de http://www.lja.mx/2018/07/aguascalientesregistra-crecimiento-economico-sostenido/

Peláez_Mejía, K. A., Payán-Quevedo, J. L., \& Salazar-Ramos, A. F. (2015). Herramienta didáctica para la explicación de conceptos de balanceo de línea en cursos de producción de los programas de ingeniería industrial. Revista Educación en ingeniería, 51-58.

Pineda, M. (01 de 03 de 2018). La industria automotriz mexicana de cara al 2018. Recuperado el 05 de 05 de 2018, de https://www.mms-

mexico.com/art\%C3\%ADculos/la-industriaautomotriz-mexicana-de-cara-al-2018

QCD Systems Inc. Website by Liquid Scope NZ. (2015). Try Z. Recuperado el 26 de 06 de 2019, de http://qcdsm.com/?cat=18

Rajadell Carreras , M., \& Sánchez García, J. L. (2010). Lean Manufacturing. La evidencia de una necesidad. España: Díaz de Santos.
Tejada-Díaz, N. L., Gisbert-Soler, V., \& PérezMolina, A. I. (Diciembre de 2017). Metodología de Estudio de Tiempo y Movimiento, Introducción al GSD. (S. Área de Innovación y Desarrollo, Ed.) 3Ciencias Empresas, 39-49. Obtenido de https://www.3ciencias.com/wpcontent/uploads/2018/01/3C-EMPRESAEspecial.pdf 